LA W RENCE LIVERMORE N A TIO NAL LABORATORY

Parametric Explosion Spectral Model

S. R. Ford, W. R. Walter

January 20, 2012 
This document was prepared as an account of work sponsored by an agency of the United States government. Neither the United States government nor Lawrence Livermore National Security, LLC, nor any of their employees makes any warranty, expressed or implied, or assumes any legal liability or responsibility for the accuracy, completeness, or usefulness of any information, apparatus, product, or process disclosed, or represents that its use would not infringe privately owned rights. Reference herein to any specific commercial product, process, or service by trade name, trademark, manufacturer, or otherwise does not necessarily constitute or imply its endorsement, recommendation, or favoring by the United States government or Lawrence Livermore National Security, LLC. The views and opinions of authors expressed herein do not necessarily state or reflect those of the United States government or Lawrence Livermore National Security, LLC, and shall not be used for advertising or product endorsement purposes.

This work performed under the auspices of the U.S. Department of Energy by Lawrence Livermore National Laboratory under Contract DE-AC52-07NA27344. 


\title{
Parametric Explosion Spectral Model
}

\author{
by Sean R. Ford and William R. Walter
}

\begin{abstract}
Small underground nuclear explosions need to be confidently detected, identified, and characterized in regions of the world where they have never before occurred. We develop a parametric model of the nuclear explosion seismic source spectrum derived from regional phases that is compatible with earthquake-based geometrical spreading and attenuation. Earthquake spectra are fit with a generalized version of the Brune spectrum, which is a three-parameter model that describes the longperiod level, corner-frequency, and spectral slope at high-frequencies. Explosion spectra can be fit with similar spectral models whose parameters are then correlated with nearsource geology and containment conditions. We observe a correlation of high gas-porosity (low-strength) with increased spectral slope. The relationship between the parametric equations and the geologic and containment conditions will assist in our physical understanding of the nuclear explosion source.
\end{abstract}

\section{Introduction}

We aim to develop a practical explosion source parametric spectral model, based on all available data, that describes nuclear explosion Pand S-wave source spectra for a variety of geologic and containment conditions. This approach follows the simple earthquake parametric spectral model based on Brune (1970), which is used for the MDAC approach (Walter and Taylor, 2001) to improve earthquake/explosion discrimination. In regions without prior explosions, the parametric model could be combined with earthquake-derived path corrections to predict explosion regional phase amplitudes, improve discriminants such as $\mathrm{P} / \mathrm{S}$ ratios, and support identification procedures (e.g., Event Classification Matrix, [ECM]) that explicitly need to use explosion discriminant probability density functions.

It is well known that depth and near-source material properties can affect seismic estimates of explosion yield, and prior work at the Nevada Test Site (NTS) (e.g., Walter et al., 1995) has found that explosions in weak materials have lower corner frequencies and steeper spectral fall-offs for P-waves than is predicted by the standard Mueller and Murphy (1971) model. As part of this research, we hope to quantify these effects as a function of frequency and wave type. Additionally, many of the most effective regional discriminants (high-frequency $\mathrm{P} / \mathrm{S}$ ratios) make use of S-waves, as do S-wave coda yield estimation techniques, yet there remain many questions about how to predict explosion S-wave amplitudes. The development of a combined P- and Swave spectral model consistent with observed regional P-and S-wave data is an objective of this work.

First, we present the data and processing and list evidence of the need for a variable spectral slope model that is dependent on the source material strength. We then fill that need via the development of a parametric spectral model with a spectral slope that is dependent on source material.

\section{Data}

We employ the NTS explosion dataset of Walter et al. (2004), specifically the raw spectra of that dataset. Waveforms are de-meaned, de-trended and instrument corrected to acceleration. The signal is windowed with a $5 \%$ cosine taper that starts before the pick, where the time before the pick is $5 \%$ of the total time window. The Fourier transform is calculated and displacement spectra are obtained via double integration in the frequency domain. Finally, the resultant amplitude spectra are interpolated and smoothed to obtain a sampling period of $0.05 \log _{10}$ $\mathrm{Hz}$.

Spectra of each seismic phase for each explosion are calculated from the recordings of stations of the Livermore NTS Network, ELK (Elko, NV), KNB (Kanab, UT), LAC (Landers, CA), and MNV (Mina, NV). The locations of these stations relative to the NTS are given in Figure 1. 


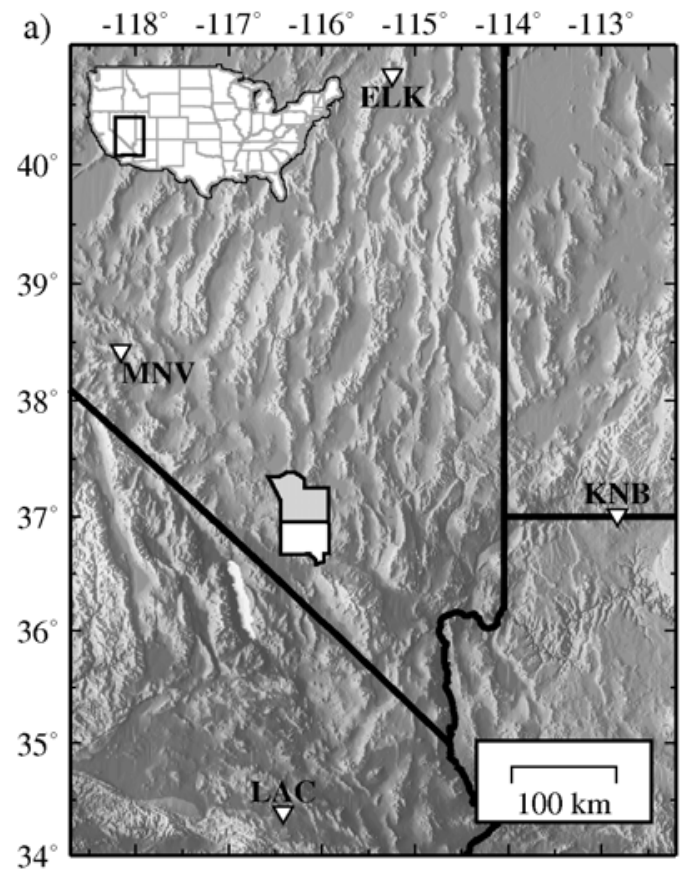

b)

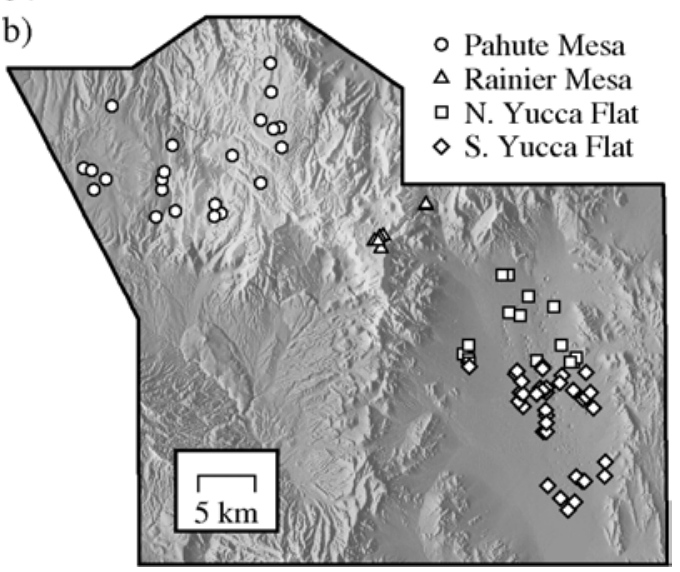

Figure 1. a) Regional map of stations used in the analysis. The location of the map within the continental US is given by the inset map. The NTS is outlined and the shaded section is shown in (b). b) Map of the northern NTS with explosion locations and Vergino and Mensing (1990) area designations.

\section{Evidence for material-dependent spectral slope}

We document the need for an explosion model with Gp-dependent spectral slope in three ways.

Evidence I: Spectral amplitude ratios (from Walter et al., 1995)
In previous work that looked at low to high frequency ratios of regional phase (e.g., Pn, Pg, Lg) amplitudes to separate explosions from earthquakes at NTS, Walter et al. (1995) noted that the results showed a strong dependence on the source media properties. Nuclear tests in weak and/or high gas porosity media tended to have higher values and discriminate better from earthquakes than explosions in stronger and/or lower gas porosity media. For example, in Figure 2 the ratio of the Lg amplitude at 1-2 $\mathrm{Hz}$ compared with the amplitude at $6-8 \mathrm{~Hz}$ is shown as a function of magnitude. The earthquakes (blue circles and green squares) show the expected trend with magnitude going from a low value for small events when the corner frequency is above $8 \mathrm{~Hz}$ and both measurements are on the constant part of the source spectra that is proportional to moment. For large magnitudes, the source corner frequency drops below $1 \mathrm{~Hz}$ and then both measures are on the part of the source spectra that decays with frequency as $\mathrm{f}^{-2}$, resulting in high spectral ratio values. As magnitude increases, the earthquakes follow a sigmoid curve as shown by the blue line, which is based on the Brune (1970) model. The explosions are split into two categories based on the source media, a high gas porosity $(\mathrm{Gp}$ ) and low strength (defined as $\rho \alpha^{2}$, where $\rho$ is density and $\alpha$ is the local compressional wave speed) group (red $\mathrm{x}$ ) and low $\mathrm{Gp}$ high strength group (black crosses), and a clear difference between the two can be seen. In fact, the low $\mathrm{Gp}$ explosions reach spectral ratio values that imply much steeper falloff than $\mathrm{f}^{-2}$.

To fit the explosion data, we used two extremes of the Denny and Goodman explosion model (1990), which has two corner frequencies. In the low Gp case we allowed the second corner to be at a higher frequency than the range of interest, giving an effective $\mathrm{f}^{-2}$ falloff. In the high Gp case we forced the corners to be the same, giving an $\mathrm{f}^{-3}$ falloff. In both cases we used the observed $3 \mathrm{~Hz}$ corner frequency of the 1993 Non-Proliferation Experiment (NPE), a kiloton chemical explosion and assumed the corner frequency scales with the cube root of $\mathrm{M}_{\mathrm{L}}(\operatorname{coda})$. Given that a pure explosion should not generate Swaves, one way to think about the Lg spectral ratio is as the product of the P-wave source ratio and a transfer function ratio, where the transfer function is a representation of how efficiently the source generated P-waves are converted (by whatever means) into S-waves. We estimated the transfer function ratio as a function of frequency as shown in Figure 2, and then multiplied the P-wave based Denny-Goodman model curves by these factors and then compared them to the Lg spectral ratio in Figure 2 . The result is a fairly reasonable first order fit to the 
a) MNV-KNB average $\mathrm{Lg}$ spectral ratios and model fits

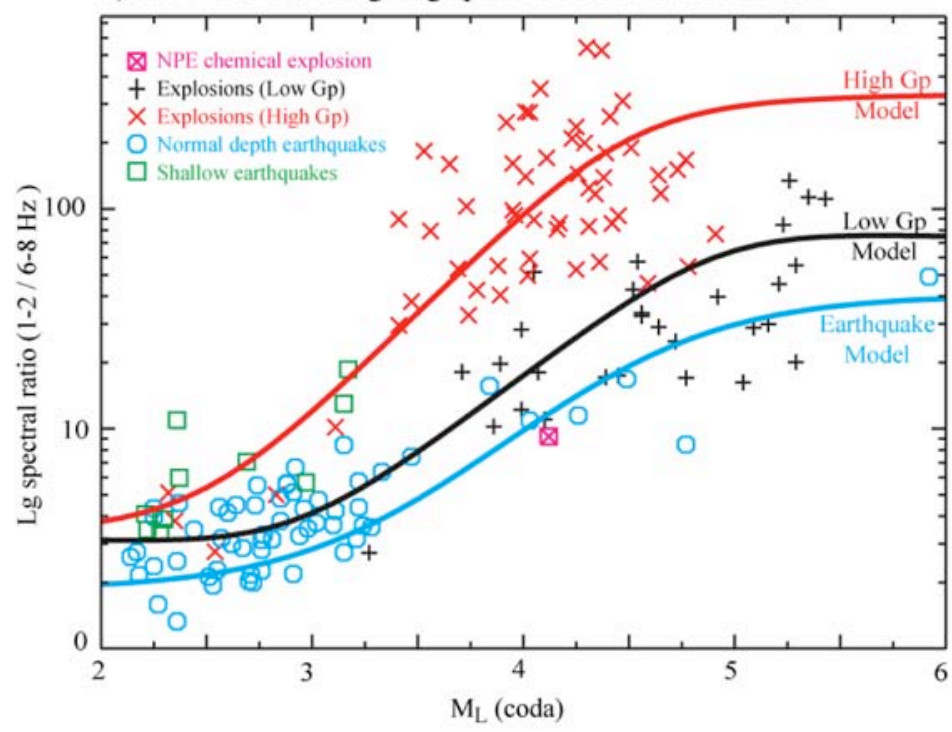

b) Source spectrum

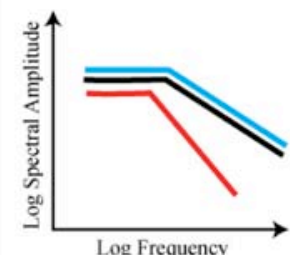

c) Lg transfer function

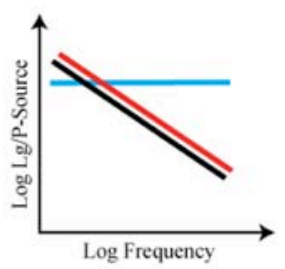

Figure 2. Simple first-order source model fits to low to high frequency spectral ratios of Lg amplitudes from Walter et al. (1995, Figure 8). Earthquake displacement spectra are fit with the Brune (1970) model (blue line), which is constant at low frequencies and falls off above a corner frequency as $\mathrm{f}^{-2}$. Explosions are fit with two extremes of the Denny and Goodman (1990) model in order to investigate explosion dependence on emplacement material (namely, gas porosity [Gp]). The 'Low Gp' model (black line) has an effective falloff similar to the earthquake model. The 'High Gp' model (red line) has a greater fall-off of $\mathrm{f}^{-}$.

data. Interestingly, since the explosion Lg transfer functions are larger than those for the earthquakes, it shifts the explosion $\mathrm{Lg}$ spectral ratios to relatively higher values than for the earthquakes, improving the discrimination performance of $\mathrm{Lg}$ spectral ratios over those of Pn alone (Walter et al. 1995, Figure 7).

While in this case, the Denny and Goodman (1990) model provides a reasonable fit to these NTS data, it is not clear how we would use it in other regions. Furthermore the limitation of only two choices of high frequency fall-off does not capture the full range of the observations. To develop a practical parametric source spectral model we want to be able to tie parameters like low-frequency level, cornerfrequency and fall-off rate to measureable properties like yield, depth, and media properties such as gas porosity, water content, and strength. In a following section we take a more general approach to fitting the NTS explosion data with a simple spectral model parameterized by a long-period level, cornerfrequency, and high-frequency roll-off.

\section{Evidence II: D\&J91-corrected spectral amplitude ratios}

Inspired by Murphy \& Bennett (2009), we analyze the Pn spectra of groups of nearby events (pairwise distance $<10 \mathrm{~km}$ ). Possible groups are shown in Figure 3. The analysis here uses the grouping marked by yellow inverted triangles in Figure 3.

These spectra are then corrected to a common source. In this case, we use an explosion with a corner frequency $\sim 1 \mathrm{~Hz}$. These parameters are estimated with the relationships given by Denny \& Johnson (1991). The spectra are then placed in one of three groups; (1) below the water table, (2) above the

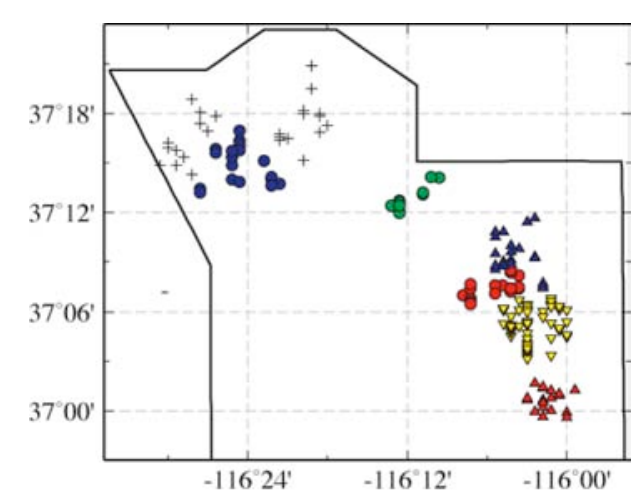

Figure 3. Possible groupings (colored symbols) of explosions at NTS (outline) where the pairwise distance between explosions is < $10 \mathrm{~km}$. The analysis here uses the group marked by the yellow inverted triangles. 

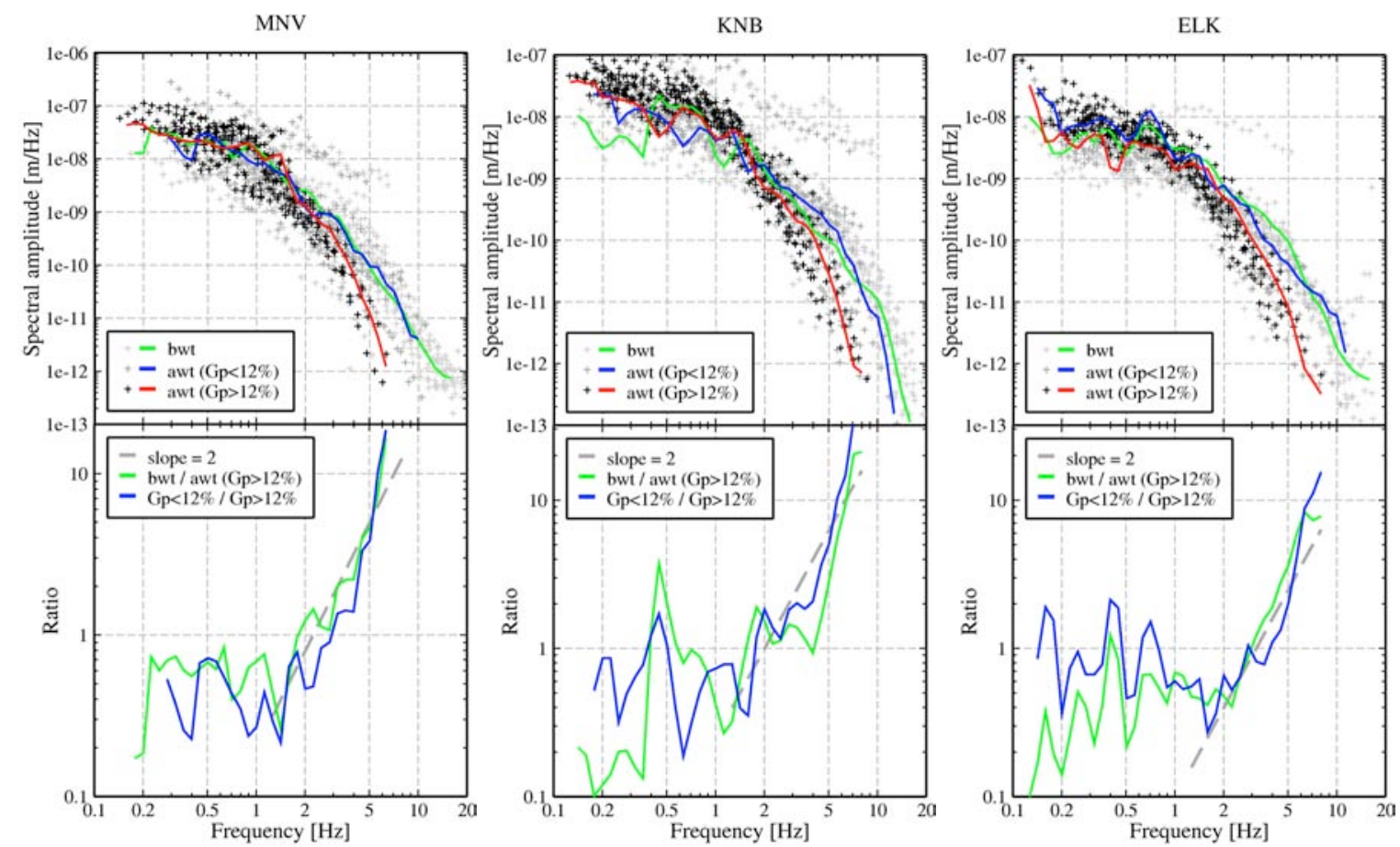

Figure 4. Pn spectral ratio analysis. First row, spectra grouped by below the water table (bwt, light gray crosses), above the water table with gas porosity $<12 \%$ (awt $\mathrm{Gp}<12 \%$, gray crosses), and above the water table with gas porosity $>12 \%$ (awt Gp $>12 \%$, black crosses). The averages of these grouped spectra are given by the colored lines. Second row, ratios of the averages are calculated. The ratios of bwt and awt $(\mathrm{Gp}<12 \%)$ to awt $(\mathrm{Gp}>12 \%)$ given by the green and blue lines, respectively, show an increased slope beginning near the corner frequency. The dashed gray line has slope of 2 and begins at $1 \mathrm{~Hz}$.

water table with gas porosity $<12 \%$, and (3) above the water table with gas porosity $>12 \%$.

We find the average spectra of each of these three groups and then calculate the ratios of those average spectra to see how spectral-slope may be different between them. The spectra, their averages and ratios are given in Figure 4. In this case, at frequencies greater than the corner there is an increase in falloff slope of about 2 for the above-thewater-table-with GP $>12 \%$ average compared to the others. This effect can be seen at MNV, KNB, and ELK. This ratio difference could be due to an increased slope of low-strength (high gas porosity) spectra as was found in the individual spectral analysis using many explosions. The difference in slope between the two populations is near 2 , as seen by the comparison with the dashed line in Figure 4.

Evidence III: Gp-dependent model analysis of variance

We also test the Gp-dependence by performing a variance analysis on a simple model,

$$
\log _{10}\left(S^{i}\right)=\beta_{1^{i}}+\beta_{2^{i}} \log _{10}(W)+\beta_{3^{i}} G p
$$

at each $\mathrm{i}^{\text {th }}$ frequency. This model is an extension of the $\mathrm{mb}(\mathrm{Pn})$ model used in Vergino and Mensing (1990), where they showed that a Gp term is required. We find $\beta_{3}$ at each $i^{\text {th }}$ frequency for the Pn spectra of 73 explosions above the water table at Yucca Flat recorded at station MNV. Figure 5a shows the parameter value at each frequency where we then correct the spectra using the average $\beta_{3}\left(\bar{\beta}_{3}=-1\right.$, gray line in Figure 5a) to obtain the model,

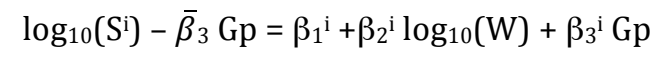

We test the significance of the third term in the model, that is the hypothesis that $\beta_{3}{ }^{\mathrm{i}}=0$, using an Ftest of the form

$$
F=\frac{\left(R S S_{1}-R S S_{2}\right) /\left(p_{2}-p_{1}\right)}{R S S_{2} /\left(n-p_{2}\right)}
$$




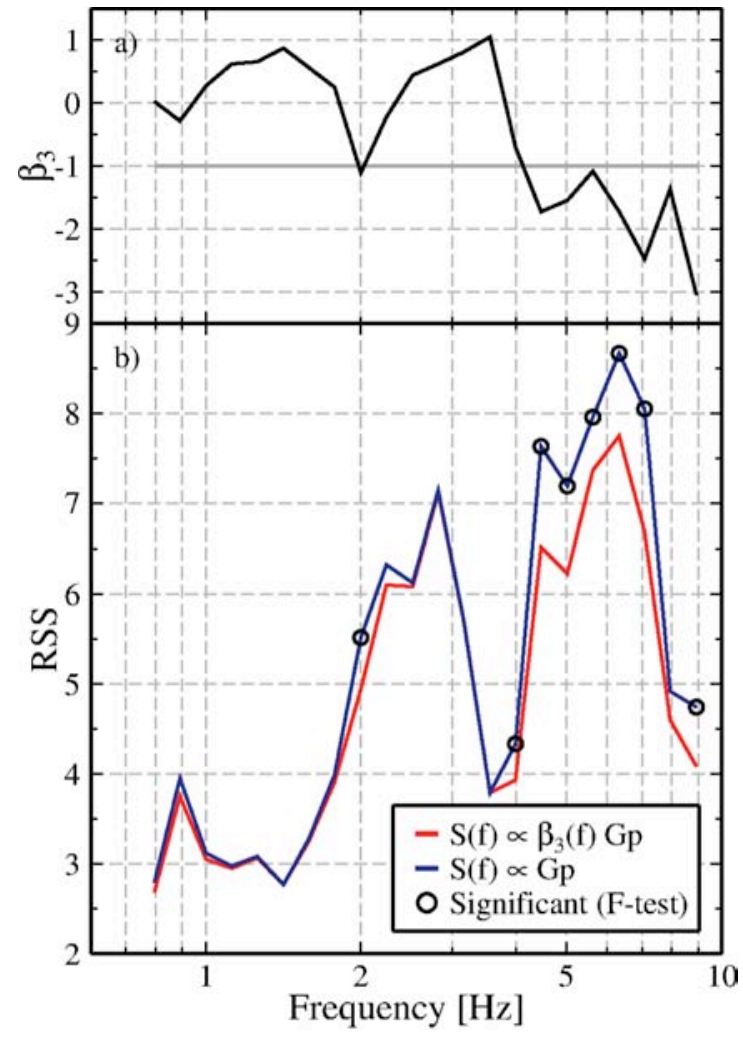

Figure 5. Gp-dependent model analysis. a) Model parameter at each frequency (black) and fixed parameter choice (gray) used in the subsequent comparison. b) Sum of the squared residuals for a model (equation (3), see text) with frequency-dependent (red) and -independent (blue) Gp using the parameters in a). The black circles mark points where the two models are significantly different.

where RSS is the sum of the squared residuals, and the subscript 2 refers to the residuals from the model in equation (4) and subscript 1 is when $\beta_{3}{ }^{i}=0$ in equation (4), $p$ is the number of parameters in the model and $\mathrm{n}$ is the number of observations. We find the $\mathrm{Gp}$ term is significant $(\mathrm{P}=0.95)$ at the high frequencies (Figure 5b), meaning an additional frequency-dependence on Gp is required.

\section{Methods}

The observed spectra are corrected for geometrical spreading and regional, frequencydependent attenuation of the form $Q=Q_{0} f \gamma$, where $Q_{0}$ is $Q$ at $1 \mathrm{~Hz}$ and $\gamma$ is the power-law dependence on frequency, f. We employ the Street et al. (1975) parametric form of geometrical spreading,

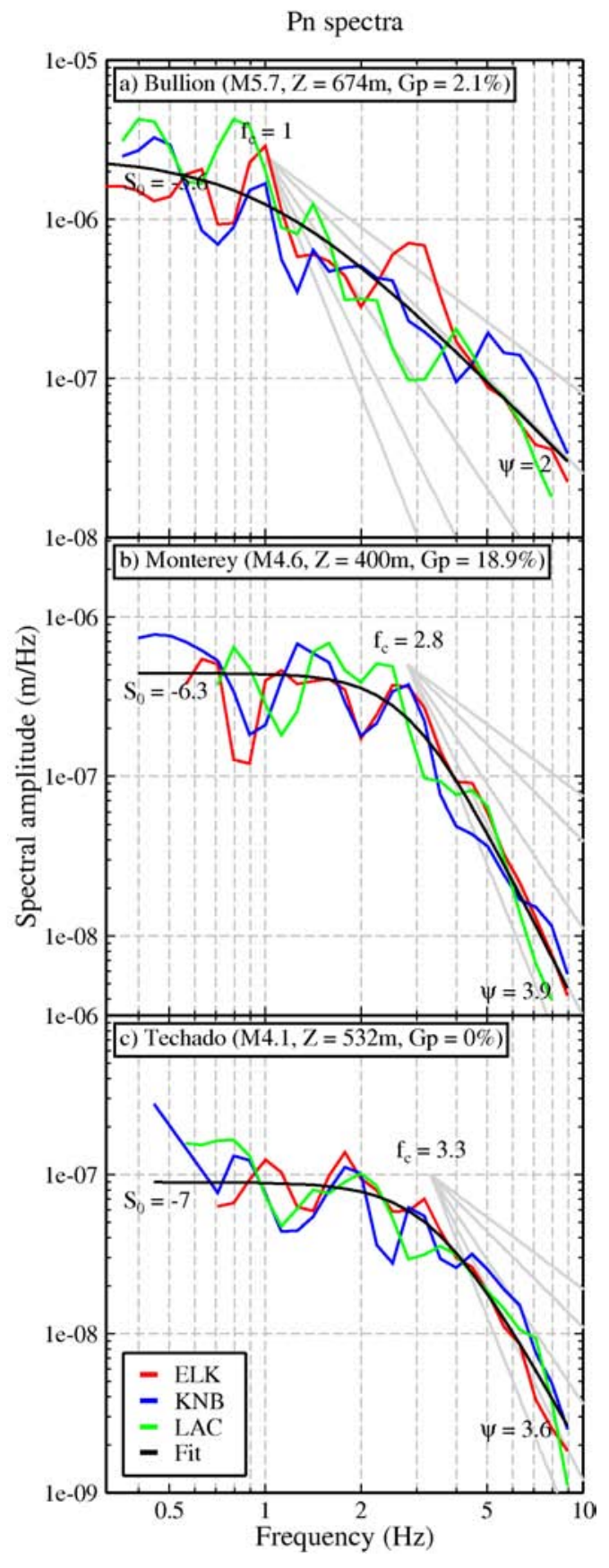

Figure 5. Pn spectra examples with the name, magnitude (where $M=m b[P n]$ ), depth (Z), and gas porosity (Gp) given in the subtitles. The longperiod level (S0), corner-frequency (fc), and falloff $(\psi)$ are given in each plot, and the spectra are colored by station and the fit is shown in the legend in the lowermost plot. a) Spectra for an event with low Gp and $\omega^{-2}$ fall-off. b) Spectra for an event with high Gp and high fall-off. c) Spectra for an event with low Gp and high fall-off. 
Table 1. NTS parameters

\begin{tabular}{lllll}
\hline Phase & $\eta$ & $r_{0}(\mathrm{~km})$ & $Q_{0}$ & $\gamma$ \\
\hline $\mathrm{Pn}$ & 1.1 & 0.001 & 210 & 0.65 \\
$\mathrm{Pg}$ & 0.5 & 100 & 190 & 0.45 \\
$\mathrm{Lg}$ & 0.5 & 100 & 200 & 0.54 \\
\hline \multicolumn{5}{r}{$r^{-1}, r<r_{0}$} \\
$G(r)=\left\{\begin{aligned} r_{0}^{-1}\left(r_{0} / r\right)^{\eta}, & r \geq r_{0}{ }^{\prime}\end{aligned}\right.$
\end{tabular}

where $r_{0}$ is the distance at which the spreading transitions from spherical- to a cylindrical-type spreading and $\eta$ is the distance dependence. The attenuation and spreading model parameters for each seismic phase are given in Table 1.

Table 2. STS parameters

\begin{tabular}{lllll}
\hline Phase & $\eta$ & $r_{0}(\mathrm{~km})$ & $Q_{0}$ & $\gamma$ \\
\hline Pn & 1.1 & 0.001 & 370 & 0.38 \\
Pg & 0.5 & 100 & 170 & 0.66 \\
Sn & 1.1 & 0.001 & 410 & 0.45 \\
Lg & 0.5 & 100 & 330 & 0.41 \\
\hline
\end{tabular}

We require three of the four stations listed above to have recorded an event with a signal-tonoise ratio greater than two. We also inspect each spectra for non-stationary noise at high-frequency (typically $>9 \mathrm{~Hz}$ ) due to multi-band recording problems and restrict spectral fitting to bands unaffected by problems. The spectra are jointly fit with a simple parametric form given by a Modified Brune spectrum with a little tighter roll-off at the corner frequency, $\mathrm{f}_{\mathrm{c}}$.

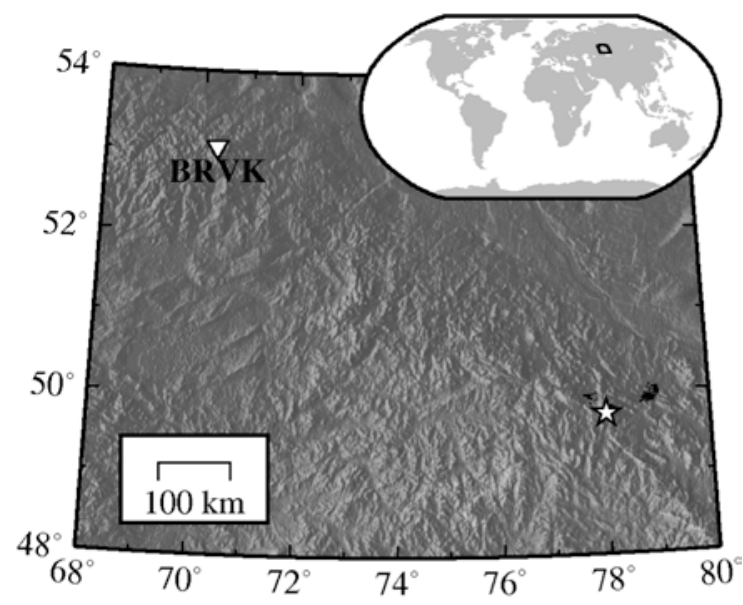

Figure 6. Regional map of station in Borovoye (BRVK) and recorded events in the archive (dots) along with the event shown in Figure 5 (star). The location of the map is given in the inset global map.
BRVK spectra (4 Oct 89 M4.7)

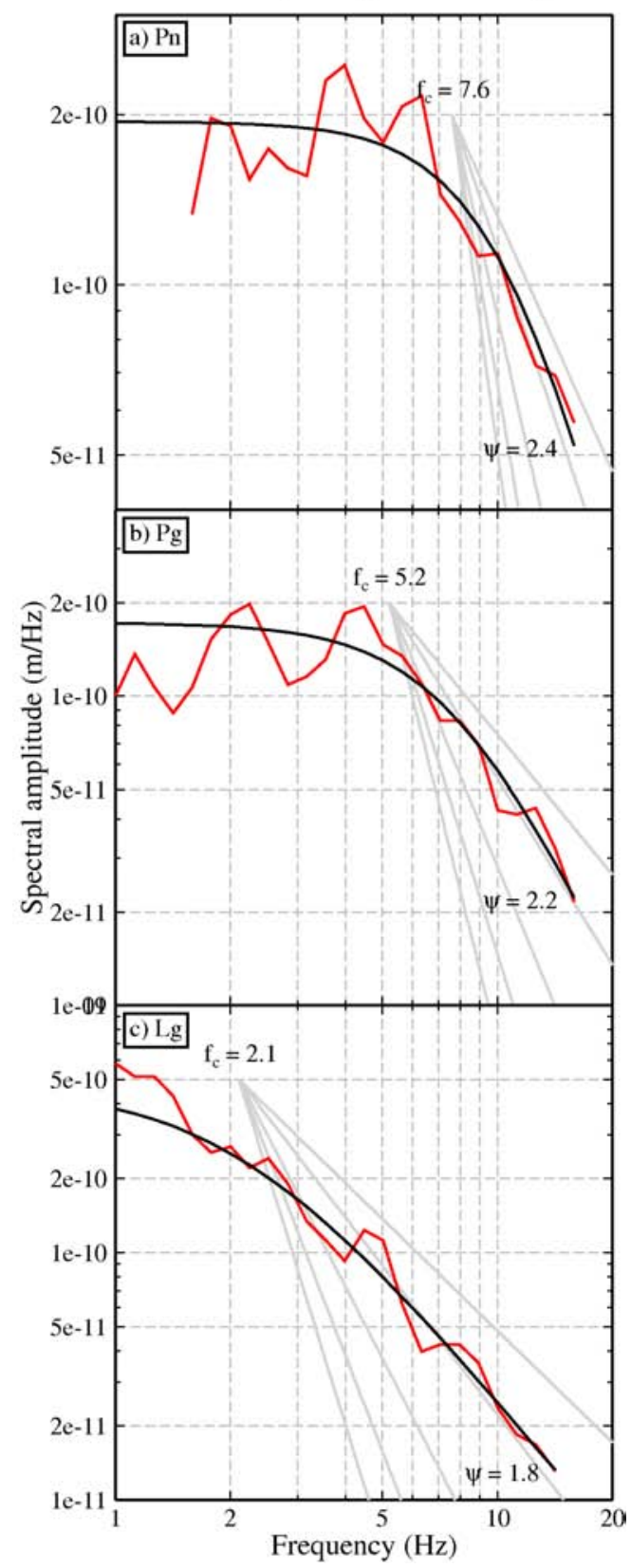

Figure 7. Pn spectra of example BRVK event (shown by star in Figure 4). Values given in each plot are defined in Figure 3.

$$
S(f)=S_{0} / \sqrt{1+\left(f^{2} / f_{c}^{2}\right)^{\psi}},
$$

in a least-squares inversion that also provides standard error for each parameter estimate. Equation (2) describes the simplest behavior expected for seismic spectra. It has a constant level at low frequencies $\left(\mathrm{S}_{0}\right)$, which is proportional to static displacement, and falls off at high frequencies with a 

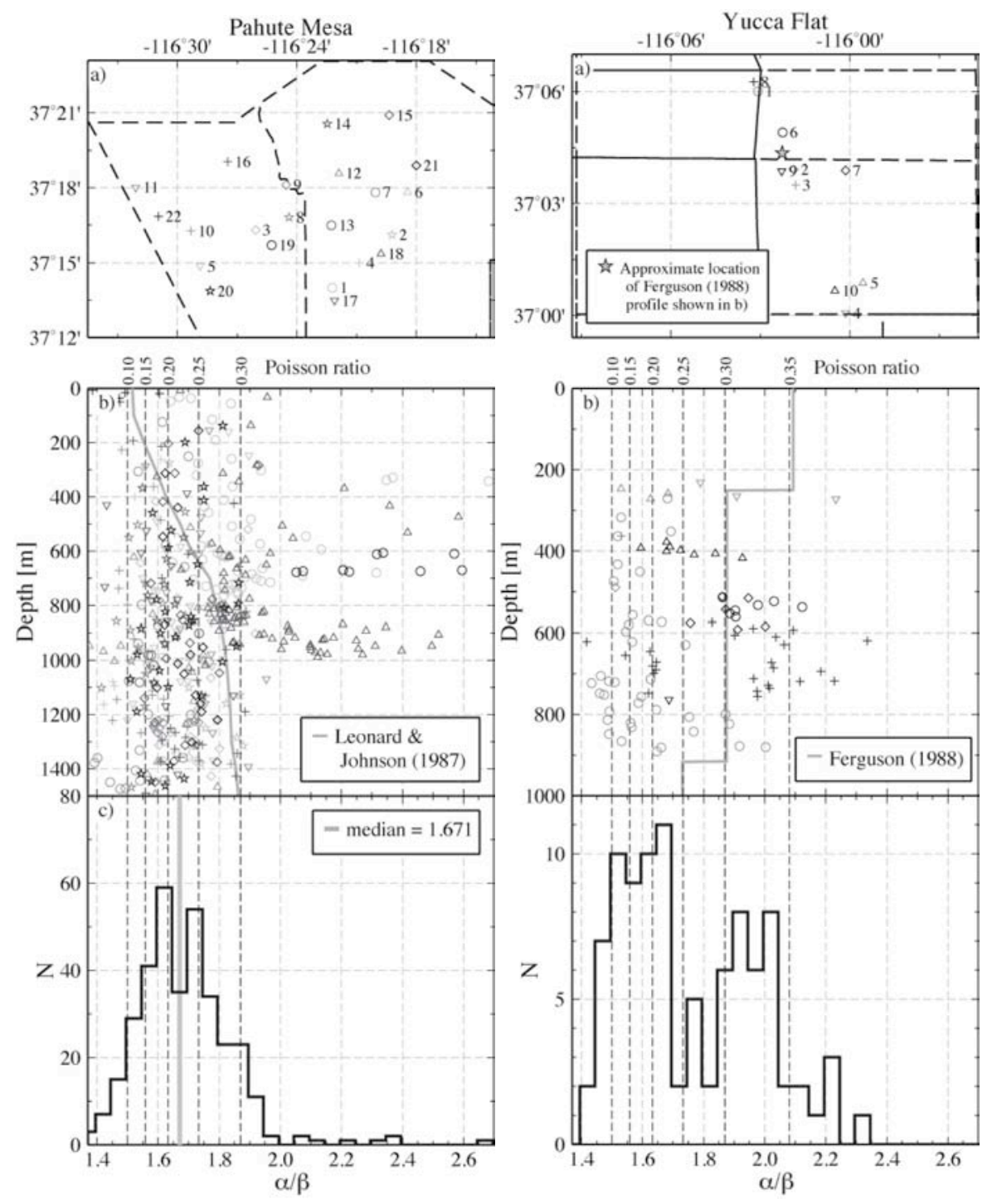

Figure 8. Analysis of Vp/Vs $(\alpha / \beta)$ ratio from USGS database (Wood, 2007) at Pahute Mesa and Yucca Flat. The ratio as a function of depth from Leonard and Johnson (1987) and Ferguson (1988) are shown for Pahute Mesa and Yucca Flat, respectively. Based on the distribution, we estimate constant Vp/Vs ratios of 1.671 and $1.871(v=0.3)$ at Pahute Mesa and Yucca Flat, respectively.

slope of $\mathrm{f}-\psi$ beyond a corner frequency $\mathrm{f}_{\mathrm{c}}$. Examples of about 200 recordings of explosions at Semipalatinsk the spectra and model fits are proportional to static Test Site of the former Soviet Union (Figure 6). As an displacement, and fall off at high frequencies with a example, we plot the regional phase spectra of one of slope of $f^{-\psi}$ beyond a corner frequency $f_{c}$. Examples of the explosions mapped in Figure 6 (shown by a star) the spectra and model fits are given in Figure 5. in Figure 7. The attenuation and spreading model BULLION (Figure 5a) is fit well with a standard $\mathrm{f}^{-2}(\psi$ parameters used to correct these spectra are given in =2) spectral fall-off, but MONTEREY (Figure 5b), Table 2. Pn parameters are from Walter and Priestley detonated in weak material, requires a steeper fall-off (1991) and Pg and Lg are from Priestley et al. (1990). where the best fit $\psi \approx 4$. We plan to complement these initial models with data

We are complementing the NTS dataset with from earthquakes in the region recorded at BRV. The the Borovoye (BRV) archive that has recently been regional phase spectra are fit with $\psi \approx 2$.

deglitched and response functions estimated The BRV regional seismic phase spectra, (Richards and Kim, 2009). This archive provides especially Pn and Pg (Figure 7a-b), show the trade-off 

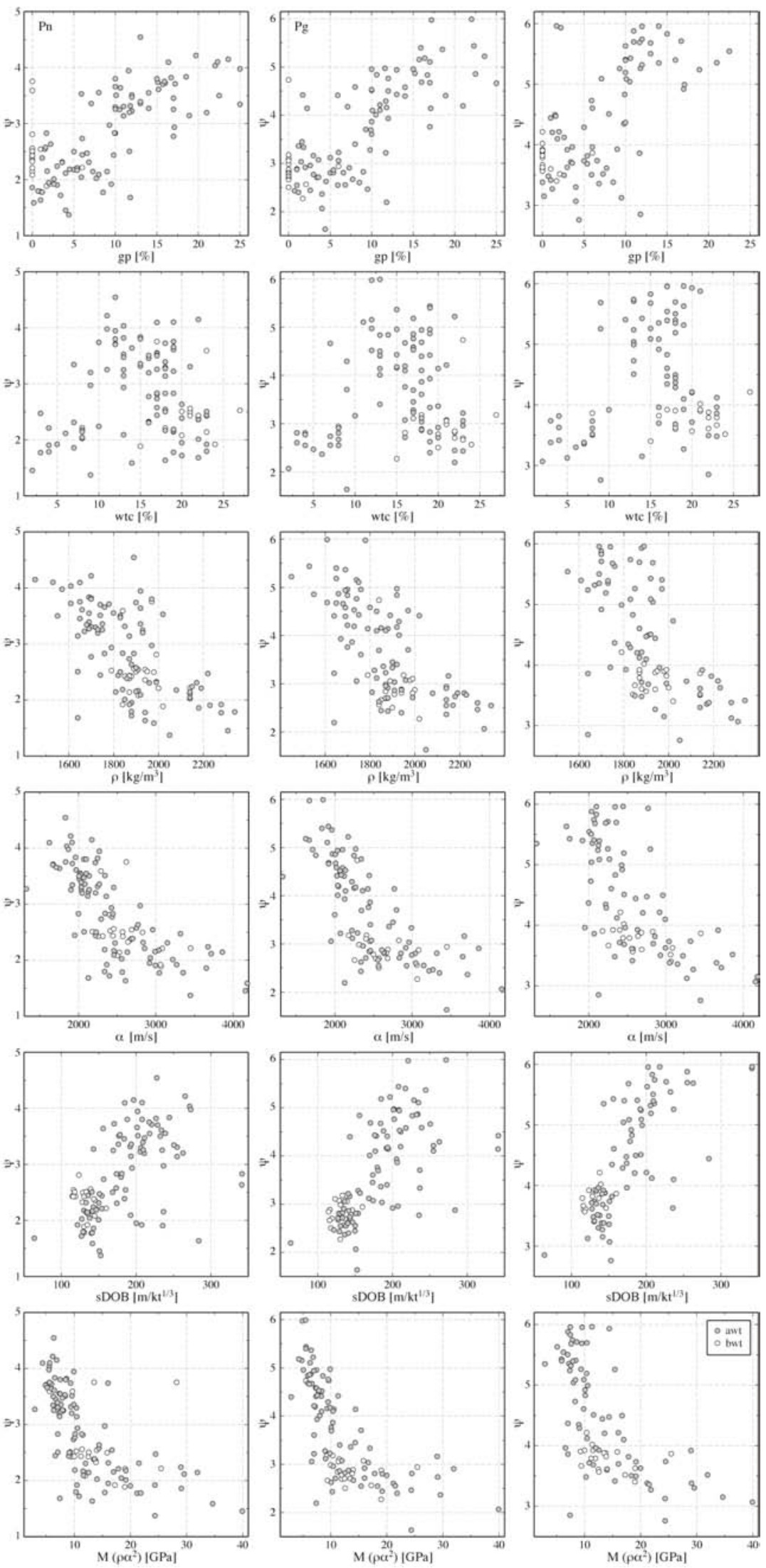

Figure 9. Spectral falloff $(\psi)$ of Pn, Pg, and Lg (1st, 2nd, and 3rd columns, respectively) versus geophysical parameters at explosion working point from Springer et al. (2002). Slight correlations can be found for gas porosity (gp), density $(\rho)$, velocity $(\alpha)$, scaled depth-ofburial (sDOB), and compressional modulus $(\mathrm{M}=$ $\rho \alpha 2$ ). Water content (wtc) shows little correlation. Explosions above and below the water table (awt and bwt, respectively) are given by the filled and open circles, respectively. The location of an explosion placed above or below the water table affects the correlation with gp, but not so much for the other parameters. The relationship of $\psi$ to $\alpha$ (and therefore also $M$ ) is more power-law than linear, or linear up to a certain value and then flat (constant $\psi$ ). The correlated parameters are used in a linear regression to find the best parametric model for $\psi$ for each seismic phase.

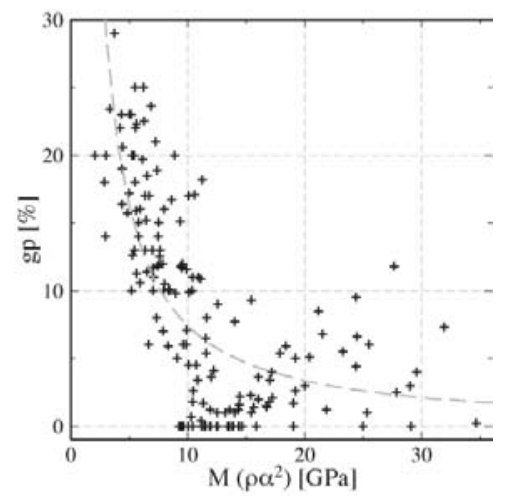

Figure 10. From Springer et al. (2002) we find a good proxy for gas porosity (gp) is a power-law relationship with compressional modulus. The dashed line is $\mathrm{gp}=$ $100 \mathrm{M}^{-1.135}$. 


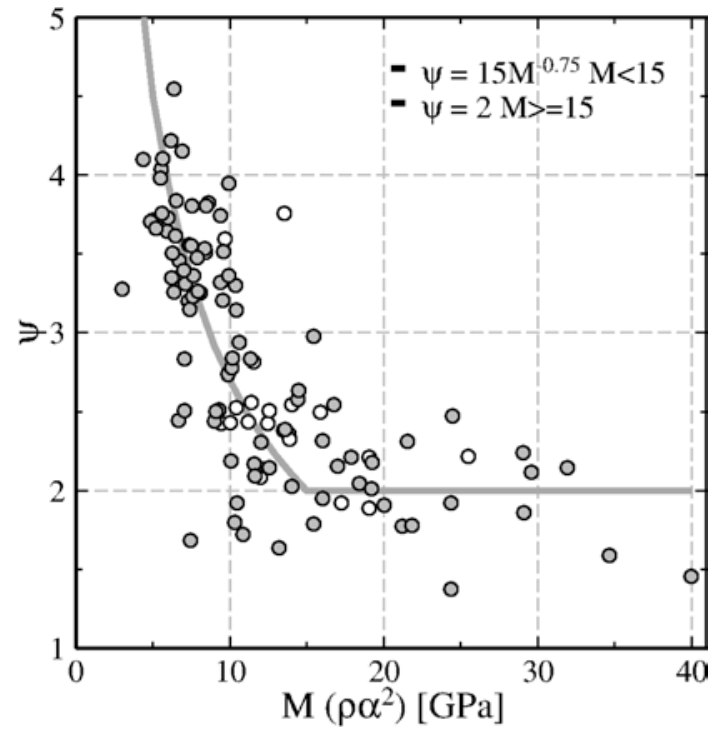

Figure 12. Spectral roll-off model.

in fitting the corner frequency and the roll-off. Attempts to diminish this trade-off will be discussed in the next section.

\section{Results}

There is a correlation between cornerfrequency and fall-off that is most probably related to the least-squares fitting of Equation (2) and not to any physical relationship. The trade-off could produce biased estimates of $\psi$, so we seek to constrain $f_{c}$ in Equation (2) using the corner frequency predicted by Denny and Johnson (1991), $\mathrm{f}_{\mathrm{c}} \mathrm{D \&}$.

In order to obtain the shear modulus and shear velocity values used in the Denny and Johnson (1991) relationships we had to convert workingpoint compressional velocities. We used the USGS database of Wood (2007) to find appropriate Vp/Vs or Poisson ratios. Figure 8 shows the analysis at Pahute Mesa, where an average $\mathrm{Vp} / \mathrm{Vs}$ ratio of 1.671 is estimated, and Yucca Flat, where an average Vp/Vs ratio of $1.871(v=0.3)$ is estimated. We set $\left.f_{c}=f_{c} D \&\right]$ in Equation (2) and estimate $\psi . \psi$ is still correlated with $\mathrm{fc}$, but it is now less due to the fitting procedure. We perform a comprehensive search for material property correlation with $\psi$, using several regressions on $\psi$ for water content $\left(S_{W}\right)$, gas porosity $(G p)$, density $(\rho)$, compressional wave speed $(\alpha)$, compressional modulus $\left(\mathrm{M}=\rho \alpha^{2}\right)$, and scaled depthof-burial $\left(\mathrm{sDOB}=\mathrm{depth} /\right.$ yield $\left.^{1 / 3}\right)$. These geophysical parameters are taken from the database of Springer et al. (2002) and are reported to represent an integrated value over the source region. Yield was inferred from $\mathrm{mb}(\mathrm{Pn})$ based on the Vergino and Mensing (1990) relationship at NTS. Figure 9 shows the dependence of $\psi$ on these parameters.

Using a step-wise regression criteria we found the most significant parameters in a linear regression model to be $M, G p$, and sDOB. Since $M$ and Gp are correlated (Figure 10), and $\psi$ is only weakly dependent on SDOB, we perform a simple leastsquares regression on $\mathrm{M}$ weighted by the estimated inverse variance of $\psi$. The model is given by the equation

$$
\psi=15 \cdot \mathrm{M}^{-3 / 4} .
$$

and the fit is shown in Figure 12. We test the fit by comparing residuals computed with a $\psi=2$ model with the model given in equation (6) by correcting all the spectra to have $\mathrm{f}_{\mathrm{c}}=1$ and calculating all high-low spectral ratios on each spectra. Figure 13 shows the mean and standard deviation of the residuals for the different models. Since they are ratios of the same spectra the absolute spectral levels are canceled out. The model with $\psi=2$ does well at the low-frequency ratios, but has a poorer fit at higher ratios where the effect of variable roll-off would be more noticeable. The model with $\psi=15 \cdot \mathrm{M}^{-3 / 4}$ dependence has lower mean residual at all ratios. The variance of the residuals is also higher for the $\psi=2$ model, suggesting that this is an effect of a fixed $\psi$ and not just a poor choice of constant $\psi$.

\section{Conclusions}

Our preliminary analysis of regional phase spectra of NTS explosions shows that a simple spectral model with variable fall-off is appropriate, and the fall-off correlates well with the material properties, gas porosity, and compressional modulus. In regions where gas porosity is unknown, the compressional modulus may be a suitable proxy. Relating fall-off $\psi$ to compressional modulus M gives the relationship in equation (6). A fit to the corrected spectra using this new model shows that improvements in high-to-low spectral ratios could allow for more robust discrimination.

\section{Acknowledgements}

We thank Terri Hauk, Stan Ruppert, Doug Dodge, and Eric Matzel for seismic analysis. This work performed under the auspices of the U.S. Department of Energy by Lawrence Livermore National Laboratory under Contract DE-AC52-07NA2734. 

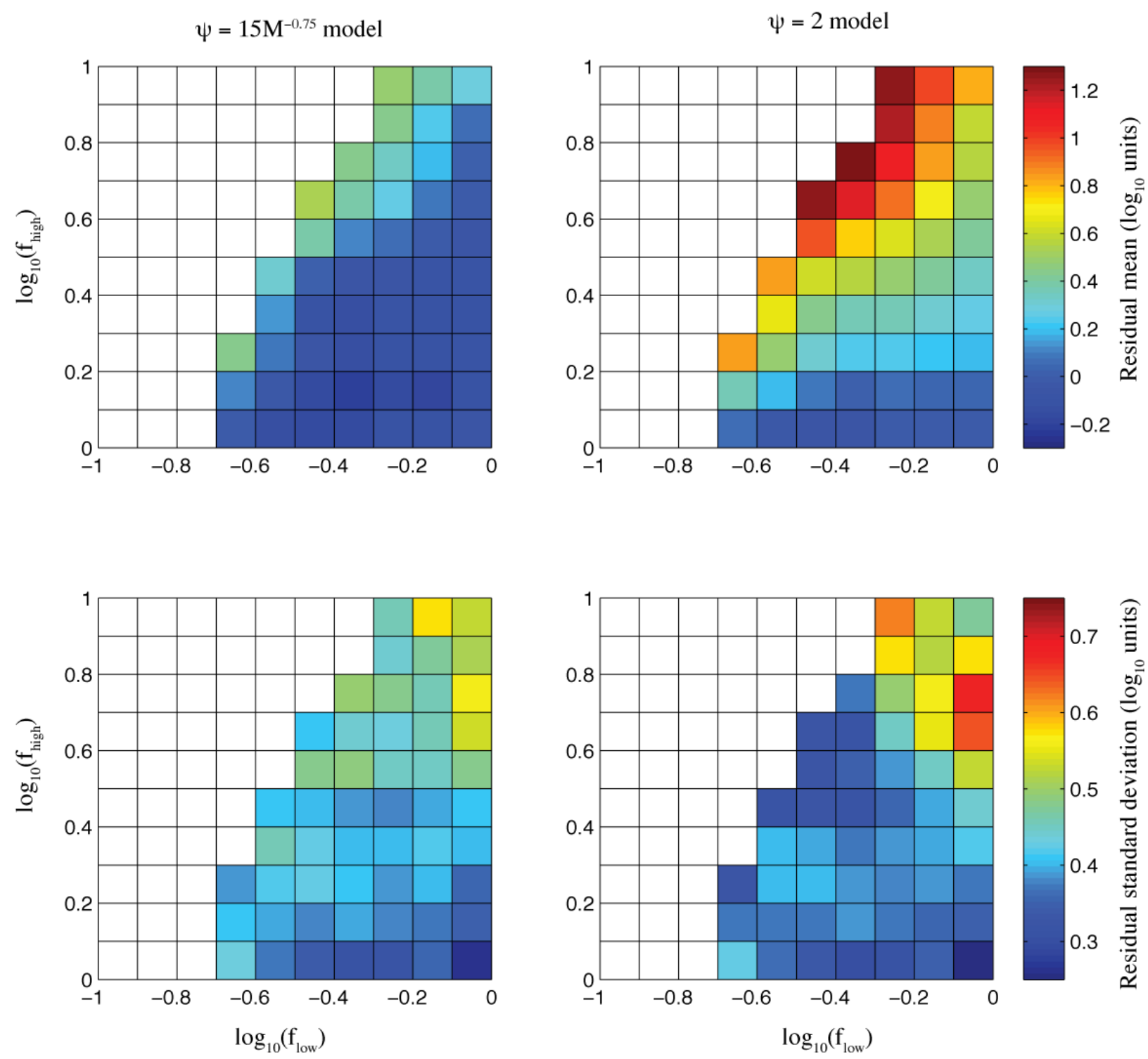

Figure 14. Mean and standard deviation of residuals due to a model with fixed roll-off ( $\psi=2$, right column) and variable roll-off that is a function of material strength (left column). Each grid point is the ratio of the high-tolow frequency spectra given by its coordinate. The spectral ratios are calculated after normalization to $f_{c}=1$ (see text for details).

\section{References}

Baker, D., W.-Y. Kim, H. Patton, G. Randall, and P. Richards (2009). Improvements to a major digital archive of seismic waveforms from nuclear explosions: The Borovoye seismogram archive, in Proceedings of the 2009 Monitoring Research Review: Ground-Based Nuclear Explosion Monitoring Technologies, LA-UR-09-05276, Vol. 1, pp. 12-21.

Brune, J. N. (1970). Tectonic stress and the spectra of seismic shear waves from earthquakes, J. Geophys. Res. 75: 4997-5009.

Denny, M. D. and D. M. Goodman (1990). A case study of the seismic source function; SALMON and STERLING reevaluated, J. Geophys. Res. 95: 19705-19723.

Denny, M. D. and L. R. Johnson (1991). The explosion seismic source function: Models and scaling laws reviewed, in Explosion Source Phenomenology, eds. Taylor S. R. et al., AGU Monograph 65: 1-24. 
Ferguson, J. F. (1988). Body-wave magnitude variation at Yucca Flat, Nevada, Bull. Seismol. Soc. Am. 78: 863872.

Fisk, M. D. (2007). Corner frequency scaling of regional seismic phases for underground nuclear explosions at the Nevada Test Site, Bull. Seismol. Soc. Am. 97: 977-988.

Ford, S. R., W. R. Walter, S. R. Ruppert, E. M. Matzel, T. F. Hauk, and M. R. Gok (2010). Toward an empiricallybased parametric explosion spectral model, in Proceedings of the 2010 Monitoring Research Review: Ground-Based Nuclear Explosion Monitoring Technologies, LA-UR-09-05276, Vol. 1, pp. 12-21.

Goldstein, P., S. P. Jarpe, K. M. Mayeda, and W. R. Walter (1994). Separation of source and propagation effects and regional distances, in Proceedings of the Symposium on the Non-Proliferation Experiment: Results and Implications for Test Ban Treaties, DOE-CONF-9404100, 6-272-6-276.

Leonard, M. A., and L. Johnson (1987). Velocity structure of Silent Canyon Caldera, Nevada Test Site, Bull. Seismol. Soc. Am. 77: 597-613.

Mueller, C. S. and J. R. Murphy (1971). Seismic characteristics of underground nuclear detonations, Part I: Seismic spectrum scaling, Bull. Seismol. Soc. Am. 61: 1675-1692.

Murphy, J. and Bennett (2010), in Proceedings of the 2010 Monitoring Research Review: Ground-Based Nuclear Explosion Monitoring Technologies, LA-UR-09-05276, Vol. 1, pp. 12-21.

Priestley, K. F., W. R. Walter, V. Martynov, and M. V. Rozhkov (1990). Regional seismic recordings of the soviet nuclear explosion of the Joint Verification Experiment, Geophys. Res. Lett. 17(2): 179-82.

Stevens, J. L. and S. M. Day (1985). The physical basis of mb:MS and variable frequency magnitude methods for earthquake/explosion discrimination, J. Geophys. Res. 90: 3009-3020.

Street, R. L., R. B. Herrmann, and O. W. Nuttli (1975). Spectral characteristics of the Lg wave generated by central United States earthquakes, Geophys. J. R. Astro. 41: 51-63.

Vergino, E. S. and R. W. Mensing (1990). Yield estimation using regional mb(Pn), Bull. Seismol. Soc. Am. 80: 656-674.

Walter, W. R, K. D. Smith, J. L. O’Boyle, T. F. Hauk, F. Ryall, S. D. Ruppert, S. C. Myers, R. Abbot, and D. A. Dodge (2004). An assembled western United States dataset for regional seismic analysis, Lawrence Livermore National Laboratory document, UCRL-TR-206630.

Walter, W. R. and S. R. Taylor (2001). A revised magnitude and distance amplitude correction (MDAC2) procedure for regional seismic discriminants, Lawrence Livermore National Laboratory document, UCRL-ID-146882.

Walter, W. R., K. M. Mayeda, and H. J. Patton (1995). Phase and spectral ratio discrimination between NTS earthquakes and explosions, Part 1: Empirical observations, Bull. Seimol. Soc. Am. 85: 1050-1067.

Walter, W. R. and K. F. Priestley (1991). Explosion Source Phenomenology. AGU monograph

Wood, D. B. (2009). Digitally available interval-specific rock-sample data compiled from historical records, Nevada Test Site and vicinity, Nye County, Nevada, USGS Data Series 297. 